\title{
DEGENERACY LOCI OF VECTOR BUNDLE MAPS AND AMPLENESS
}

\author{
JØRGEN ANDERS GEERTSEN
}

\section{Introduction}

Let $X$ be an algebraic variety and $E, F$ vector bundles on $X$, of ranks $e, f$ respectively. For any vector bundle map $\phi: E \rightarrow F$ and $k \in \mathrm{N}, 0 \leq k \leq$ $\min \{e, f\}$, the $k^{\prime}$ th degeneracy locus of $\phi$ is the set

$$
X_{k}(\phi):=\{x \in X \mid \operatorname{rank} \phi(x) \leq k\} .
$$

Since a linear map has rank $\leq k$ if and only if all $k+1$-minors vanish, the set $X_{k}(\phi)$ can also be described as the zero locus of the section $\wedge^{k+1} \phi \in$ $\Gamma\left(X,\left(\wedge^{k+1} E\right)^{*} \otimes \wedge^{k+1} F\right)$. As such, it comes with a natural structure as a closed subscheme of $X$.

Degeneracy loci turn up in a number of geometric constructions. For instance, the common zero locus of a finite set of sections of a vector bundle is a degeneracy locus. In Brill-Noether Theory, degeneracy loci show up as the subsets of the Jacobian which parametrizes complete linear systems of a specific degree and dimension bounded below.

For a map $\phi: E \rightarrow F$, the expected dimension of $X_{k}(\phi)$ is the number $\operatorname{dim} X-(e-k)(f-k)$. We denote this number by $m_{k}$ throughout this paper. Then we have, for any $\phi: E \rightarrow F$,

$$
\text { If } X_{k}(\phi) \neq \emptyset \quad \text { then } \operatorname{dim} X_{k}(\phi) \geq m_{k} .
$$

For instance, if $E=\mathscr{O}_{X}$ is trivial and $k=0$, then $\phi: E \rightarrow F$ can be viewed as a section $\phi \in \Gamma(X, F)$. Then $X_{0}(\phi)$ is equal to $Z(\phi)$, the zero-locus of the section $\phi$, and in this case the expected dimension of $Z(\phi)$ is $\operatorname{dim} X-f$. This is just Krull's Hauptidealsatz: $Z(\phi)$ is locally cut out by $f$ equations, so its codimension in $X$ should be $f$.

Now it might very well be the case that $n o \phi: E \rightarrow F$ has $X_{k}(\phi)$ with the expected dimension. For example on $\mathrm{P}^{2}$, the bundle $E=\mathscr{O} \oplus \mathscr{O}(1)$ has rank 2 so one expects a generic section to vanish in codimension 2. But most sections

Received January 4, 1999. 
don't vanish at all, namely those of the form $(\lambda, \sigma)$ where $\lambda \in k \backslash\{0\}$, and the other sections vanish too much, namely on a line, i.e. in codimension 1 . So even for very nice globally generated bundles it can happen that no $X_{k}(\phi)$ has the 'right' dimension. We define the number $t_{k}=t_{k}(X, E, F)$ as follows:

$$
t_{k}:=\min \left\{\operatorname{dim} X_{k}(\phi) \mid \phi: E \rightarrow F \text { a morphism with } X_{k}(\phi) \neq \emptyset\right\} .
$$

Note that since we can take for $\phi$ the zero-morphism $0: E \rightarrow F$, which has $X_{k}(0)=X$ for all $k$, the number $t_{k}$ is well-defined and $t_{k} \leq \operatorname{dim} X$. Also, the inequality $m_{k} \leq t_{k}$ holds for all $k(0 \leq k \leq \min \{e, f\}$ as always $)$. For instance, in the above example, $m_{0}=0$ and $t_{0}=1$.

We are concerned with the following relative situation. Suppose $E, F$ are vector bundles on $X$ as above. Let $Y$ be an irreducible algebraic variety and suppose $f: X \rightarrow Y$ is a proper surjective map. We say that the bundle $E^{*} \otimes F$ is ample relative to $f$, written 'rel $f$ ', if it is ample on all fibers of $f$, i.e. the restriction $\left.E^{*} \otimes F\right|_{f^{-1}(y)}$ is an ample vector bundle for all (closed) points $y \in Y$. Our purpose is to describe what happens to the images of the degeneracy loci under the map $f$. The results are the following.

THEOREM 1. Suppose $X$ is a complex algebraic variety and $E, F$ are vector bundles on $X$. Let $f: X \rightarrow Y$ be a proper surjective map to another variety $Y$, and suppose $E^{*} \otimes F$ is ample rel $f$. Suppose furthermore that $E^{*} \otimes F$ is globally generated.

Let $\phi: E \rightarrow F$ be any vector bundle map. Then

(1) If $t_{k} \leq \operatorname{dim} Y$, then for any irreducible component $Z \subset f\left(X_{k}(\phi)\right)$ we have $\operatorname{dim} Z \geq t_{k}$.

(2) If $t_{k}>\operatorname{dim} Y$, then $f\left(X_{k}(\phi)\right)=Y$.

(3) Furthermore, if $m_{k} \leq \operatorname{dim} Y$, then $t_{k} \leq \operatorname{dim} Y$. If $m_{k}=\operatorname{dim} Y$, then $f\left(X_{k}(\phi)\right)=Y$.

REMARK 1. In case $X_{k}(\phi)$ is empty, statement (1) is inconclusive since there are no irreducible components $Z$ to choose from. However, statement (2) says that $X_{k}(\phi)$ must necessarily be non-empty if $t_{k}>\operatorname{dim} Y$, since its image under $f$ is equal to $Y$. Similarly, statement (3) says that all $X_{k}(\phi)$ must be non-empty if $m_{k}=\operatorname{dim} Y$. These results are sharp: If for instance $t_{k}=\operatorname{dim} Y$ but $m_{k}<\operatorname{dim} Y$, one cannot assert that all degeneracy loci are non-empty. For example, take the bundle $F:=\mathscr{O}(1) \oplus \mathscr{O}(1)$ on $\mathrm{P}^{1}$ and look at zero-sets of sections of $F$. Then $t_{0}=0, m_{0}=-1$ and $F$ has a nowhere vanishing section.

The relations of Theorem 1 with theorems in the litterature are as follows. Taking $Y=\mathrm{pt}$, we are in the situation of an ample vector bundle $E^{*} \otimes F$ on a complete variety $X$. In this case statement (1) is trivial. Statement (2) and 
(3) however combine to the existence result of Fulton and Lazarsfeld [4] (for globally generated bundles). Fulton and Lazarsfeld's result says that if $m_{k} \geq 0$, then for any $\phi: E \rightarrow F$, we have $X_{k}(\phi) \neq \emptyset$. If $m_{k}>0$ then also $t_{k}>0$ so this statement follows from (2). If $m_{k}=0=\operatorname{dim} Y$, the statement follows from (3).

Again taking $Y=\mathrm{pt}$, the statement in (3) " $m_{k} \leq \operatorname{dim} Y \Rightarrow t_{k} \leq \operatorname{dim} Y$ " means in this case that $m_{k} \leq 0 \Rightarrow t_{k}=0$, since we always have $t_{k} \geq 0$. For instance, if $E=\mathscr{O}_{X}$ and we look at zero-schemes of sections of the bundle $F$ then $m_{0}=\operatorname{dim} X-\operatorname{rank} F$ and the statement is the following geometric observation:

Corollary 2. Suppose $X$ is a projective variety and $F$ an ample, globally generated vector bundle on $X$ with $\operatorname{rank} F \geq \operatorname{dim} X$. Then there is a section $\sigma \in \Gamma(X, F)$ such that $Z(\sigma)$ is a finite non-empty set of points.

This in turn gives an easy proof of a special case of the Bloch-Gieseker theorem [2] which states that for an ample vector bundle $F$ on a projective variety $X$, if rank $F \geq n:=\operatorname{dim} X$, then $c_{n}(F)>0$. In fact, letting $C=$ $\{\sigma \in \Gamma(X, F) \mid Z(\sigma) \neq \emptyset\}$ be the cone of sections that do vanish somewhere, and $l$ the length of the zero subscheme of a generic section in $C$ (which is well-defined by the corollary) then borrowing some of the ideas behind the proof of Theorem 1 yields the following formula for $c_{n}(F)$ :

$$
c_{n}(F)=l \cdot \operatorname{deg} C .
$$

(Here $\operatorname{deg} C$ is the degree of the cone $C$ ). This formula gives a new lower bound for $c_{n}(F)$ valid for all ample and globally generated vector bundles $F$ (Corollary 13). The formula also implies the funny result that if $c_{n}(F)$ happens to be a prime number, and rank $F>n$, then $F$ has a section $\sigma$ such that $Z(\sigma)$ consists of exactly one (reduced) point (Corollary 12). In general, if $F$ is "sufficiently positive", one expects $l=1$ - for instance, this holds if $F(-1)$ is globally generated for some very ample line bundle $\mathscr{O}(1)$ on $X$ (Example 7).

If on the other hand one takes $Y=X$ and $f=1$ in Theorem 1 then there is no ampleness condition on $E^{*} \otimes F$ and statement (1) must hold, i.e. $\operatorname{dim} Z \geq t_{k}$ for any irreducible component $Z$ of $X_{k}(\phi)$. This shows that for bundles $E, F$ such that $E^{*} \otimes F$ is globally generated the number $t_{k}$ could equally well be defined as the minimum dimension of any irreducible component $Z$ in any $X_{k}(\phi)$.

Statements (1) and (2) imply that for any $\phi: E \rightarrow F$, if $Z$ denotes an irreducible component of $f\left(X_{k}(\phi)\right)$, then $\operatorname{dim} Z \geq \min \left\{\operatorname{dim} Y, t_{k}\right\}$. Since $t_{k} \geq$ $m_{k}$, this implies the weaker statement

$$
\operatorname{dim} Z \geq \min \left\{\operatorname{dim} Y, m_{k}\right\} .
$$


This is the Theorem of Steffen [11, Th. 0.3] (for globally generated bundles). However, Steffens algebraic methods are completely different from ours. In fact, our Theorem 1 arose from trying to give a simple geometric proof of Steffens result. The underlying geometric idea of our approach does not use global generation, but to get a uniform statement like Theorem 1 we must have $E^{*} \otimes F$ globally generated. On the other hand, we obtain a stronger result than Steffens for this class of bundles, and the statement in Theorem 1 is optimal (see Example 4). Furthermore we can (almost) work in arbitrary characteristic: if one assumes that the map $f$ in Theorem 1 is generically smooth (which is of course guaranteed in char. 0 ) then the proof goes through over an arbitrary algebraically closed ground field.

ACKNOWLEDGEMENTS. I am indebted to R. Lazarsfeld, A. Hirschowitz and J. Hansen for many suggestions and valuable conversations. Several of the ideas in section 5 developed during discussions with L. Manivel at Institut J. Fourier in Grenoble, France, and I thank Manivel and the Institut for inviting me to Grenoble.

\section{Ample vector bundles}

Let $X$ be an algebraic scheme over the algebraically closed ground field $K$ and $E$ a vector bundle on $X$. Recall that by definition, $E$ is ample if for all coherent sheaves $\mathscr{F}$ on $X$ there exists an integer $N \in \mathrm{N}$ such that

$$
n \geq N \Rightarrow \mathscr{F} \otimes \operatorname{Sym}^{n} E \quad \text { is globally generated. }
$$

Denote by $\mathrm{P}(E)$ the projective bundle of lines in $E$. Then $E$ is ample on $X$ if and only if the tautological line bundle $\mathscr{O}_{\mathrm{P}\left(E^{*}\right)}(1)$ is ample on $\mathrm{P}\left(E^{*}\right)$ [7]. Next suppose $Y$ is another scheme and $f: X \rightarrow Y$ is a map. We say $E$ is ample relative to $f$ (written 'rel $f$ ') if there is an open affine covering $Y=\cup U_{\alpha}$ such that for all $\alpha$, the bundle $\left.E\right|_{f^{-1}\left(U_{\alpha}\right)}$ is an ample vector bundle. If we let $q: \mathrm{P}\left(E^{*}\right) \rightarrow X$ denote the projection map, then this condition can be translated as follows: $E$ is ample rel $f$ if and only if $\mathscr{O}_{\mathrm{P}\left(E^{*}\right)}(1)$ is ample rel $f \circ q$.

If $f$ is a proper map, the condition of relative ampleness is equivalent to the following property [11, Lemma 1.5]: For all coherent sheaves $\mathscr{F}$ on $X$ there exists $N \in \mathrm{N}$ such that

$$
n \geq N \Rightarrow R^{i} f_{*}\left(\mathscr{F} \otimes \operatorname{Sym}^{n} E\right)=0, \quad i>0 .
$$

If $Y$ is a point, this is simply Serre Vanishing, since then $R^{i} f_{*}\left(\mathscr{F} \otimes \operatorname{Sym}^{n} E\right)=$ $H^{i}\left(X, \mathscr{F} \otimes \operatorname{Sym}^{n} E\right)$. 
Of course if $E$ is ample relative to a map $f$, then $E$ is ample on the fibers of $f$, these being closed subschemes of $X$. Conversely, if $f$ is a proper map, this condition is also sufficient: $E$ is ample rel $f$ if and only if for all (closed) points $y \in Y$, the restriction $\left.E\right|_{f^{-1}(y)}$ is an ample vector bundle [7, Prop. 4.4]. It is this very explicit description of relative ampleness that we shall use.

ExAmple 1. Let $\pi: \mathrm{Bl}_{\mathrm{pt}}\left(\mathrm{P}^{2}\right) \rightarrow \mathrm{P}^{2}$ be the blow-up of the projective plane at a point. If $E$ denotes the exceptional divisor, then $\mathscr{O}_{X}(-E)$ is ample rel $\pi$. Because on the fiber $E=\mathrm{P}^{1}$ it is $\mathscr{O}_{\mathrm{Pl}}(1)$ which is ample, and all other fibers are points so there is no condition. For the same reason, $\mathscr{O}_{X}(E)$ isn't ample $\operatorname{rel} \pi$.

EXAmple 2. Consider

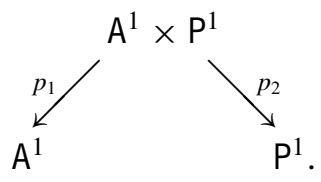

Then $p_{2}^{*} \mathscr{O}(1)$ is ample rel $p_{1}$ because on the fibers $\mathrm{P}^{1}$ it's just $\mathscr{O}_{\mathrm{Pl}}(1)$. Since $p_{2}$ is an affine morphism, $p_{2}^{*} \mathscr{O}(1)$ is also ample rel $p_{2}$.

The following lemma will be used in the proof of Theorem 1 .

Lemma 3. Suppose $f: F^{\prime} \rightarrow F$ is a birational map of positive dimensional complete varieties and $E$ an ample vector bundle on $F$. Then

$$
H^{0}\left(F^{\prime}, f^{*} E^{*}\right)=0 .
$$

Proof. To begin, note that the morphism $f$ is automatically proper. Indeed, $F$ is a variety, hence separated over $K$, and the composition $F^{\prime} \rightarrow F \rightarrow K$ is proper.

We first prove the lemma in case $E=\mathscr{L}$ is a line bundle. Assume $\sigma \in$ $H^{0}\left(F^{\prime}, f^{*} \mathscr{L}^{*}\right)$ is a non-zero section. Then $c_{1}\left(f^{*} \mathscr{L}\right) \cap\left[F^{\prime}\right]=-[Z(\sigma)]$; hence

$$
\begin{aligned}
\int_{F^{\prime}} c_{1}\left(f^{*} \mathscr{L}\right)^{\operatorname{dim} F^{\prime}} \cap\left[F^{\prime}\right] & =-\int_{F^{\prime}} c_{1}\left(f^{*} \mathscr{L}\right)^{\operatorname{dim} F^{\prime}-1} \cap[Z(\sigma)] \\
& =-\int_{F} c_{1}(\mathscr{L})^{\operatorname{dim} F^{\prime}-1} \cap f_{*}[Z(\sigma)] \\
& \leq 0
\end{aligned}
$$

by the projection formula and Nakai's criterion for ampleness. But since $f$ is birational, $f_{*}\left[F^{\prime}\right]=[F]$ and $\operatorname{dim} F^{\prime}=\operatorname{dim} F$, so again by Nakai's criterion 
and the projection formula,

$$
\int_{F^{\prime}} c_{1}\left(f^{*} \mathscr{L}\right)^{\operatorname{dim} F^{\prime}} \cap\left[F^{\prime}\right]=\int_{F} c_{1}(\mathscr{L})^{\operatorname{dim} F} \cap[F]>0 ;
$$

a contradiction. What we have done is basically to observe that since $\mathscr{L}$ is big and nef and these notions are birationally invariant, the pull-back $f^{*} \mathscr{L}$ is also big and nef, hence the dual doesn't have sections.

Now let $E$ be an ample vector bundle of arbitrary rank on $F$. Suppose to the contrary that $0 \neq \sigma \in H^{0}\left(F^{\prime}, f^{*} E^{*}\right)$ is a non-zero section. Then the map $f^{*} E \stackrel{\sigma^{*}}{\longrightarrow} \mathscr{O}_{F^{\prime}}$ is non-zero. Choose an ample line bundle $\mathscr{L}$ on $F$-for example, the determinant of $E$ will do. Pick $n \in \mathrm{N}$ so $\mathscr{L}^{*} \otimes \operatorname{Sym}^{n} E$ is generated by its global sections. Then also $f^{*} \mathscr{L}^{*} \otimes \operatorname{Sym}^{n} f^{*} E$ is globally generated. Hence there is a surjection

$$
\mathscr{O}_{F^{\prime}}^{\oplus l} \longrightarrow f^{*} \mathscr{L}^{*} \otimes \operatorname{Sym}^{n} f^{*} E \longrightarrow 0 .
$$

Since the map $f^{*} E \stackrel{\sigma^{*}}{\longrightarrow} \mathscr{O}_{F^{\prime}}$ is non-zero, also the symmetric power map $\operatorname{Sym}^{n} f^{*} E \stackrel{\operatorname{Sym}^{n} \sigma^{*}}{\longrightarrow} \mathscr{O}_{F^{\prime}}$ is non-zero. Tensoring with $f^{*} \mathscr{L}^{*}$ we see that the map

$$
f^{*} \mathscr{L}^{*} \otimes \operatorname{Sym}^{n} f^{*} E \stackrel{1 \otimes \operatorname{Sym}^{n} \sigma^{*}}{\longrightarrow} f^{*} \mathscr{L}^{*}
$$

is non zero. Comparing with $(*)$, we conclude that the composition

$$
\mathscr{O}_{F^{\prime}}^{\oplus l} \longrightarrow f^{*} \mathscr{L}^{*} \otimes \operatorname{Sym}^{n} f^{*} E \longrightarrow f^{*} \mathscr{L}^{*}
$$

is non-zero. But this means that $H^{0}\left(F^{\prime}, f^{*} \mathscr{L}^{*}\right) \neq 0$, in contradiction with the first part of the proof. This concludes the proof of Lemma 3.

Remark 2. The conclusion of the lemma holds under the weaker assumption that $\operatorname{dim} F=\operatorname{dim} F^{\prime}$ and $f$ is any dominant (=surjective) map, not neccesarily birational. All we have to do in the proof of Lemma 3 is to change the equality $f_{*}\left[F^{\prime}\right]=[F]$ to $f_{*}\left[F^{\prime}\right]=d[F]$ where $d>0$ is some positive integer. Then the exact same proof applies.

\section{Degeneracy loci}

Let $X$ be an algebraic scheme and $E, F$ vector bundles on $X$ of ranks $e, f$ respectively. Suppose $\phi: E \rightarrow F$ is a map. The $k^{\prime}$ th degeneracy locus of $\phi$ is the set

$$
X_{k}(\phi):=\{x \in X \mid \operatorname{rank} \phi(x) \leq k\} .
$$

This is a closed subscheme of $X$, given locally by the vanishing of the $k+1$ minors of $\phi$. To analyze the degeneracy loci $X_{k}(\phi)$ we use the following 
construction. Put $k^{\prime}=e-k$ and consider $\mathrm{G}:=G_{k^{\prime}}(E)$, the grassmanian bundle of $k^{\prime}$-planes in $E$ with its natural projection map $\pi: G_{k^{\prime}}(E) \longrightarrow X$.

On $\mathrm{G}$ we have the tautological exact sequence

$$
0 \longrightarrow \mathscr{S} \longrightarrow \pi^{*} E \longrightarrow \mathscr{Q} \longrightarrow 0
$$

where $\mathscr{S}, \mathscr{Q}$ are the tautological sub- and quotient bundles respectively. We denote a point in $\mathrm{G}$ by $\left(x, H_{x}\right) ; H_{x}$ is a $k^{\prime}$-plane in the vector space $E(x)$. The fiber of $\mathscr{S}$ over the point $\left(x, H_{x}\right) \in \mathrm{G}$ is just the $k^{\prime}$-plane $H_{x} \subset E(x)$, and the fiber of $\mathscr{Q}$ over $\left(x, H_{x}\right)$ is $E(x) / H_{x}$. Consider the composition:

$$
\sigma: \mathscr{S} \hookrightarrow \pi^{*} E \stackrel{\pi^{*} \phi}{\longrightarrow} \pi^{*} F .
$$

Then $Z(\sigma)=\left\{\left(x, H_{x}\right) \mid H_{x} \subset \operatorname{ker} \phi(x)\right\}$. So $Z(\sigma)$ maps onto $X_{k}(\phi)$ by $\pi$, because the map $\phi(x): E(x) \rightarrow F(x)$ has rank $\leq k$ if and only if the kernel is at least $k^{\prime}$-dimensional, i.e. contains some $k^{\prime}$-plane. The fiber of the restriction $\bar{\pi}: Z(\sigma) \longrightarrow X_{k}(\phi)$ over the point $x \in X_{k}(\phi)$ is just the grassmanian of $k^{\prime}$-planes contained in $\operatorname{ker} \phi(x)$. If $x \in X_{k}(\phi) \backslash X_{k-1}(\phi)$, i.e. the rank of $\phi(x)$ is exactly $k$, then $\operatorname{dim} \operatorname{ker} \phi(x)=k^{\prime}$ and $\bar{\pi}^{-1}(x)=\{\operatorname{ker} \phi(x)\}$ is a point. In other words, in case $X_{k}(\phi) \backslash X_{k-1}(\phi) \neq \emptyset, \bar{\pi}$ is birational.

The idea of the grassmanian construction is to reduce questions on general degeneracy loci to questions on zero-schemes of sections of vector bundles. This is achieved by constructing the section $\sigma \in \Gamma\left(\mathrm{G}, \mathscr{S}^{*} \otimes \pi^{*} F\right)$ whose zeroscheme maps onto $X_{k}(\phi)$ and in good cases even is birational to $X_{k}(\phi)$. Then we would like to transport the information we have on $E^{*} \otimes F$ to information on $\mathscr{S}^{*} \otimes \pi^{*} F$ :

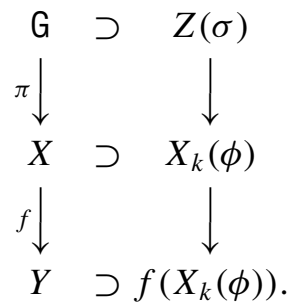

In the setting of Theorem 1 , we know that $E^{*} \otimes F$ is ample on the fibers of $f$. So we would like to have $\mathscr{S}^{*} \otimes \pi^{*} F$ ample on the fibers of $f \circ \pi$, in particular $\mathscr{S}^{*} \otimes \pi^{*} F$ would then have to be ample on the fibers of $\pi$. But on these fibers

$$
\left.\mathscr{S}^{*} \otimes \pi^{*} F\right|_{\pi^{-1}(\mathrm{pt})}=\overbrace{\left.\left.\left.\mathscr{S}^{*}\right|_{\pi^{-1}(\mathrm{pt})} \oplus \mathscr{S}^{*}\right|_{\pi^{-1}(\mathrm{pt})} \oplus \ldots \oplus \mathscr{S}^{*}\right|_{\pi^{-1}(\mathrm{pt})}}^{f}
$$

so we are really asking for the ampleness of $\left.\mathscr{S}^{*}\right|_{\pi^{-1}(\mathrm{pt})}$. However, $\pi^{-1}(\mathrm{pt})$ is just the ususal grassmanian $\mathrm{G}=G_{k^{\prime}}(E(\mathrm{pt}))$ of $k^{\prime}$-planes in the vector space $E(\mathrm{pt})$ 
and $\left.\mathscr{S}^{*}\right|_{\pi^{-1}(\mathrm{pt})}$ is just the dual of the tautological subbundle on $\mathrm{G}$. Unfortunately this bundle is almost never ample, as the following proposition (which surely belongs to folklore) shows.

Proposition 4. Let $V$ be a finite dimensional vector space and $G:=G_{k}(V)$ the grassmanian of $k$-planes in $V$. Let $\mathscr{S}$ be the tautological subbundle of $\mathrm{G} \times V$. Then $\mathscr{S}^{*}$ is ample $\Leftrightarrow k=1$.

Proof. If $k=1, \mathrm{G}=\mathrm{P}(V)$ and $\mathscr{S}^{*}=\mathscr{O}(1)$ is ample.

If $k>1$, pick a $k-1$-dimensional subspace $W \subset V$ and put

$$
\mathrm{P}:=\{H \in \mathrm{G} \mid H \supset W\} \cong \mathrm{P}(V / W) .
$$

Then $\mathrm{P}=\mathrm{P}^{n-k}$ is a projective space (here $n=\operatorname{dim} V$ ). For $x \in \mathrm{P}$, denote by $l_{x}$ the line in $V / W$ corresponding to $x$. Then it is clear that $\left.\mathscr{S}\right|_{\mathrm{P}}(x)=l_{x} \oplus W$, hence $\left.\mathscr{S}\right|_{\mathrm{P}}=\mathscr{O}_{\mathrm{P}}(-1) \oplus \mathscr{O}_{\mathrm{P}}^{\oplus k-1}$. So $\left.\mathscr{S}^{*}\right|_{\mathrm{P}}=\mathscr{O}_{\mathrm{P}}(1) \oplus \mathscr{O}_{\mathrm{P}}^{\oplus k-1}$ which isn't ample for $k>1$.

However, the key to prove Theorem 1 turns out to be the fact that the dual of a certain vector bundle does not have any non-zero global sections, and for ample vector bundles this property is preserved under birational maps (Lemma 3 ) even though the property of amplitude itself is not preserved.

Suppose now that the bundle $E^{*} \otimes F$ is globally generated, on the algebraic variety $X$. Let $V \subset \Gamma\left(X, E^{*} \otimes F\right)$ be a finite dimensional vector space of sections that generate $E^{*} \otimes F$. Consider the projection:

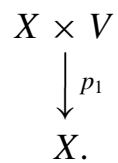

There is a tautological map $\tau: p_{1}^{*} E \rightarrow p_{1}^{*} F$ defined by

$$
\begin{gathered}
\tau(x, \phi): p_{1}^{*} E(x, \phi) \longrightarrow p_{1}^{*} F(x, \phi) \\
E(x) \stackrel{\phi(x)}{\longrightarrow} F(x) .
\end{gathered}
$$

Alternatively, let $\tau_{t}$ be the tautological section of $p_{1}^{*}\left(\mathscr{O}_{X} \otimes V\right)$ on $X \times V$; then $\tau$ is just the image of $\tau_{t}$ under the (surjective) map $p_{1}^{*}\left(\mathscr{O}_{X} \otimes V\right) \rightarrow p_{1}^{*}\left(E^{*} \otimes F\right)$. Denote by $D_{k}(\tau)$ the $k^{\prime}$ th degeneracy locus of $\tau$. Then by definition $D_{k}(\tau)=$ $\{(x, \phi) \mid \operatorname{rank} \phi(x) \leq k\}=\left\{(x, \phi) \mid x \in X_{k}(\phi)\right\}$.

We can apply the grassmanian construction described earlier to the map $\tau: p_{1}^{*} E \rightarrow p_{1}^{*} F$ on the scheme $X \times V$. Hence we consider the grassmanian 
$\mathrm{G}=G_{k^{\prime}}\left(p_{1}^{*} E\right)$ with its projection map $\pi$ to $X \times V$. We have the tautological exact sequence on $\mathrm{G}$ :

$$
0 \longrightarrow \mathscr{S} \longrightarrow \pi^{*} p_{1}^{*} E \longrightarrow \mathscr{Q} \longrightarrow 0,
$$

and the section $\sigma \in \Gamma\left(\mathrm{G}, \mathscr{S}^{*} \otimes \pi^{*} p_{1}^{*} F\right)$ defined as the composition

$$
\sigma: \mathscr{S} \hookrightarrow \pi^{*} p_{1}^{*} E \stackrel{\pi^{*} \tau}{\longrightarrow} \pi^{*} p_{1}^{*} F .
$$

The crucial point is:

Proposition 5. $\sigma$ is a regular section of $\mathscr{S}^{*} \otimes \pi^{*} p_{1}^{*} F$ and $Z(\sigma)$ is an irreducible variety, of dimension $\operatorname{dim} X+\operatorname{dim} V-(e-k)(f-k)$.

PRoof. To prove the proposition we use another description of $\sigma$ and then employ a general result (Lemma 6 ) on locally free sheaves and their associated vector bundles. First note that $G_{k^{\prime}}\left(p_{1}^{*} E\right)$ identifies with $G_{k^{\prime}}(E) \times V$ and that we have a commutative square

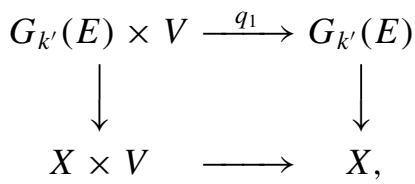

where $q_{1}$ is the projection on the first factor. We denote the natural map $G_{k^{\prime}}(E) \rightarrow X$ by $\pi^{\prime}$. Let $\mathscr{S}^{\prime}, \mathscr{Q}^{\prime}$ denote the tautological sub- and quotient bundles on $G_{k^{\prime}}(E)$; under $q_{1}$ these bundles pull back to $\mathscr{S}$ and $\mathscr{Q}$. On $X$ we have the exact sequence

$$
\mathscr{O}_{X} \otimes V \longrightarrow E^{*} \otimes F \longrightarrow 0,
$$

hence on $G_{k^{\prime}}(E)$ the following exact diagram

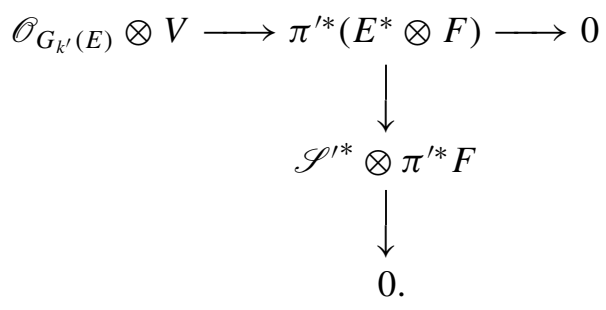

Denote the kernel of the composition by $\mathscr{K}$ :

$$
0 \longrightarrow \mathscr{K} \longrightarrow \mathscr{O}_{G_{k^{\prime}}(E)} \otimes V \longrightarrow \mathscr{S}^{\prime *} \otimes \pi^{* *} F \longrightarrow 0 .
$$

Then $\mathscr{K}$ is a locally free sheaf of rank $\operatorname{dim} V-(e-k) f$ on $G_{k^{\prime}}(E)$. Viewing $\mathrm{G}$ as $G_{k^{\prime}}(E) \times V$ amounts to considering it as the total space of the locally free sheaf 
$\mathscr{O}_{G_{k^{\prime}}(E)} \otimes V$ on $G_{k^{\prime}}(E)$, and the tautological section $\sigma_{t} \in \Gamma\left(\mathrm{G}, q_{1}^{*}\left(\mathscr{O}_{G_{k^{\prime}}(E)} \otimes V\right)\right)$ is simply the pull-back via $\pi$ of the tautological section $\tau_{t} \in \Gamma\left(X \times V, p_{1}^{*}\left(\mathscr{O}_{X} \otimes\right.\right.$ $V))$. Now $\sigma$ is defined as the image of $\pi^{*} \tau$ in $\Gamma\left(\mathrm{G}, \mathscr{S}^{*} \otimes \pi^{*} p_{1}^{*} F\right)$, hence as the image of $\pi^{*} \tau_{t} \in \Gamma\left(\mathrm{G}, \pi^{*} p_{1}^{*}\left(\mathscr{O}_{X} \otimes V\right)\right)$ in $\Gamma\left(\mathrm{G}, \mathscr{S}^{*} \otimes \pi^{*} p_{1}^{*} F\right)$ via the composition $\pi^{*} p_{1}^{*}\left(\mathscr{O}_{X} \otimes V\right) \rightarrow \pi^{*} p_{1}^{*}\left(E^{*} \otimes F\right) \rightarrow \mathscr{S}^{*} \otimes \pi^{*} p_{1}^{*} F$. Viewing $\pi^{*} \tau_{t}=\sigma_{t}$ as a section of $\pi^{*} p_{1}^{*}\left(\mathscr{O}_{X} \otimes V\right)=q_{1}^{*}\left(\mathscr{O}_{G_{k^{\prime}}(E)} \otimes V\right)$ and noting $\mathscr{S}^{*} \otimes \pi^{*} p_{1}^{*} F=q_{1}^{*}\left(S^{\prime *} \otimes \pi^{\prime *} F\right)$, this means that $\sigma$ is the image of $\sigma_{t}$ under the $\operatorname{map} q_{1}^{*}\left(\mathscr{O}_{G_{k^{\prime}}(E)} \otimes V\right) \rightarrow q_{1}^{*}\left(S^{*} \otimes \pi^{* *} F\right)$. Now the total space Spec Sym $\mathscr{K}^{*}$ of $\mathscr{K}$ is a subscheme of $\operatorname{Spec} \operatorname{Sym}\left(\mathscr{O}_{G_{k^{\prime}}(E)} \otimes V\right)=\mathrm{G}$. The proposition will certainly follow if we show that Spec $\operatorname{Sym} \mathscr{K}^{*}=Z(\sigma)$, because any closed embedding of an affine space bundle in another is always regular, and the dimension of $\operatorname{Spec} \operatorname{Sym} \mathscr{K}^{*}$ is $\operatorname{dim} G_{k^{\prime}}(E)+\operatorname{rank} \mathscr{K}=\operatorname{dim} X+(e-k) k+$ $\operatorname{dim} V-(e-k) f=\operatorname{dim} X+\operatorname{dim} V-(e-k)(f-k)$. Now $\operatorname{Spec} \operatorname{Sym} \mathscr{K}^{*}=$ $Z(\sigma)$ follows from the next general observation, suggested by Anders Thorup:

Lemma 6. Let $T$ be a scheme and

$$
0 \longrightarrow \mathscr{H}^{\prime \prime} \longrightarrow \mathscr{H} \longrightarrow \mathscr{H}^{\prime} \longrightarrow 0
$$

an exact sequence of locally free sheaves on T. Let $H=\operatorname{Spec} \operatorname{Sym} \mathscr{H}^{*}$ be the total space associated to $\mathscr{H}$ and $p: H \rightarrow T$ the projection map. Let $\sigma_{t} \in \Gamma\left(H, p^{*} \mathscr{H}\right)$ be the tautological section of $p^{*} \mathscr{H}$ and $\sigma \in \Gamma\left(H, p^{*} \mathscr{H}^{\prime}\right)$ its image global section. Then as schemes,

$$
Z(\sigma)=\operatorname{Spec} \operatorname{Sym} \mathscr{H}^{\prime \prime *} .
$$

Proof. We show that the corresponding functor of points are equal, see [10, Prop. 1, Chap. II.6]. Hence let $Q$ be a scheme. We must show that

$$
\operatorname{Hom}(Q, Z(\sigma))=\operatorname{Hom}\left(Q, \operatorname{Spec} \operatorname{Sym} \mathscr{H}^{\prime \prime *}\right) \text {. }
$$

Now by the universal property of $Z(\sigma)$, to give a morphism from $Q$ to $Z(\sigma)$ is the same thing as giving a morphism $f: Q \rightarrow H$ such that $f^{*} \sigma=0$. But to give a morphism $f: Q \rightarrow H$ is equivalent to give a map $g=p \circ f: Q \rightarrow T$ + a section of $g^{*} \mathscr{H}$ (namely $f^{*} \sigma_{t}$ ). Hence we see that

$$
\begin{aligned}
\operatorname{Hom}(Q, Z(\sigma))= & \text { morphisms } g: Q \rightarrow T+\text { a section } s \text { of } g^{*} \mathscr{H} \\
& \text { such that the image of } s \text { is } 0 \text { in } \Gamma\left(Q, g^{*} \mathscr{H}^{\prime}\right) .
\end{aligned}
$$

But the kernel of the map $\Gamma\left(Q, g^{*} \mathscr{H}\right) \rightarrow \Gamma\left(Q, g^{*} \mathscr{H}^{\prime}\right)$ is $\Gamma\left(Q, g^{*} \mathscr{H}^{\prime \prime}\right)$. Hence

$$
\begin{aligned}
\operatorname{Hom}(Q, Z(\sigma)) & =\text { morphisms } g: Q \rightarrow T+\text { a section of } g^{*} \mathscr{H}^{\prime \prime} \\
& =\operatorname{Hom}\left(Q, \operatorname{Spec} \operatorname{Sym} \mathscr{H}^{\prime \prime *}\right) .
\end{aligned}
$$


This proves Lemma 6.

The fact that $\sigma$ is a regular section is important for the following reason. Suppose $T$ is any scheme and $\mathscr{H}$ is any locally free sheaf on $T$, of rank $r$. Let $\sigma \in \Gamma(T, \mathscr{H})$ be a section. Then there is a Koszul resolution corresponding to $\sigma$ :

$$
0 \rightarrow \wedge^{r} \mathscr{H}^{*} \rightarrow \wedge^{r-1} \mathscr{H}^{*} \rightarrow \cdots \rightarrow \mathscr{H}^{*} \rightarrow \mathscr{I} \rightarrow 0
$$

where $\mathscr{I}$ is the ideal sheaf of $Z(\sigma)$. Now $\sigma$ is a regular section of $\mathscr{H}$ if and only if this resolution is exact. By restricting to $Z(\sigma)$ one sees that in this case, $\left.\mathscr{H}^{*}\right|_{Z(\sigma)} \cong \mathscr{I} / \mathscr{I}^{2}$, hence that the normal sheaf $\mathscr{N}_{Z(\sigma) / T}=\left.\mathscr{H}\right|_{Z(\sigma)}$ is locally free, of rank $r$.

\section{Degeneracy loci and amplitude}

In this section we prove

THEOREM 7. Suppose $X$ is a complex algebraic variety and $E, F$ are vector bundles on $X$. Let $f: X \rightarrow Y$ be a proper surjective map to another variety $Y$, and suppose $E^{*} \otimes F$ is ample rel $f$. Suppose furthermore that $E^{*} \otimes F$ is globally generated.

Let $\phi: E \rightarrow F$ be any vector bundle map. Then

(1) If $t_{k} \leq \operatorname{dim} Y$, then for any irreducible component $Z \subset f\left(X_{k}(\phi)\right)$ we have $\operatorname{dim} Z \geq t_{k}$.

(2) If $t_{k}>\operatorname{dim} Y$, then $f\left(X_{k}(\phi)\right)=Y$.

(3) Furthermore, if $m_{k} \leq \operatorname{dim} Y$, then $t_{k} \leq \operatorname{dim} Y$. If $m_{k}=\operatorname{dim} Y$, then $f\left(X_{k}(\phi)\right)=Y$.

To motivate the proof, let's sketch the idea in the simplest case where $E=\mathscr{O}_{X}$ and $k=0$. Then degeneracy loci of morphisms $\phi: \mathscr{O}_{X} \rightarrow F$ are just zero sets of sections $\phi \in \Gamma(X, F)$. Suppose $\phi \in \Gamma(X, F)$ is a regular section so that in particular the dimension of $Z(\phi)$ is the expected $\operatorname{dim} X-\operatorname{rank} F$. Then $t_{0}=\operatorname{dim} X-\operatorname{rank} F$ and the real problem is to prove statement (1), i.e. that if $\operatorname{dim} X-\operatorname{rank} F \leq \operatorname{dim} Y$ then $Z(\phi)$ doesn't drop dimension under $f$. Suppose to the contrary that $\operatorname{dim} f(Z(\phi))<\operatorname{dim} Z(\phi)$. Then restricting the map of normal sheaves $\mathscr{N}_{Z(\phi) / X} \rightarrow f^{*} \mathscr{N}_{f(Z(\phi)) / Y}$ to a positive dimensional fiber one gets a map to a trivial bundle. Since $\phi$ is regular, $\mathscr{N}_{Z(\phi) / X}=\left.F\right|_{Z(\phi)}$, so the map must be zero since $F$ is ample on the fibers of $f$. On the other hand, if $p \in Z(\phi)$ is chosen suitably the tangent map $T_{X}(p) \rightarrow T_{Y}(f(p))$ is surjective and also $T_{Y}(f(p)) \rightarrow \mathscr{N}_{f(Z(\phi)) / Y}(f(p))$ is surjective. Hence the 
composition $T_{X}(p) \rightarrow \mathscr{N}_{f(Z(\phi)) / Y}(f(p))$ is surjective. But in the diagram

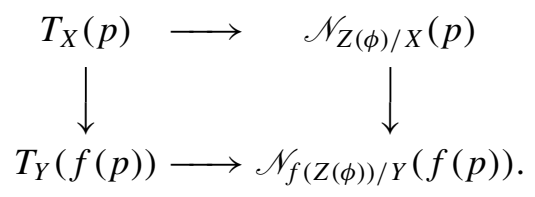

the vertical right hand map is zero, which is a contradiction.

This idea of proof uses that $\phi$ is a regular section of $F$. In general it is not clear whether $F$ has any regular sections at all! But we can always construct the regular section $\sigma$ as in Prop. 5, and it is in this setting the above idea will be applied.

Proof. Let $\phi: E \rightarrow F$ be any map and pick a finite dimensional vector space $V \subset \Gamma\left(X, E^{*} \otimes F\right)$ that generates $E^{*} \otimes F$ and such that $\phi \in V$.

We can consider the tautological map $\tau: p_{1}^{*} E \rightarrow p_{1}^{*} F$ on $X \times V$ as described on the half page before Proposition 5. Recall that $\tau$ is defined as

$$
\begin{gathered}
\tau(x, \phi): p_{1}^{*} E(x, \phi) \longrightarrow p_{1}^{*} F(x, \phi) \\
E(x) \stackrel{\phi(x)}{\longrightarrow} F(x) .
\end{gathered}
$$

The degeneracy locus $D_{k}(\tau)$ is given by $D_{k}(\tau)=\left\{(x, \phi) \mid x \in X_{k}(\phi)\right\}$. Consider the diagram

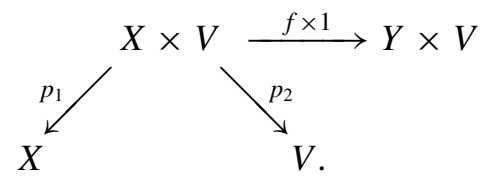

Then $f \times 1$ is a proper surjective map, and $p_{1}^{*}\left(E^{*} \otimes F\right)$ is ample on the fibers of $f \times 1$. Put $\mathscr{W}:=\overline{p_{2}\left(D_{k}(\tau)\right)} \subset V$ (i.e. $\mathscr{W}$ is the Zariski closure of the image of $D_{k}(\tau)$ by $\left.p_{2}\right)$ and let $\overline{p_{2}}: D_{k}(\tau) \rightarrow \mathscr{W}$ denote the restriction. Then the fiber ${\overline{p_{2}}}^{-1}(\psi) \cong X_{k}(\psi)$ so the generic fiber dimension is equal to $t_{k}$ by definition of $t_{k}$ and semi-continuity of fiber dimension. Hence

$$
t_{k}=\operatorname{dim} D_{k}(\tau)-\operatorname{dim} \mathscr{W}=m_{k}+\operatorname{dim} V-\operatorname{dim} \mathscr{W}
$$

where as usual $m_{k}=\operatorname{dim} X-(e-k)(f-k)$. Consider

$$
f \times 1\left(D_{k}(\tau)\right)=\left\{(y, \phi) \mid y \in f\left(X_{k}(\phi)\right)\right\} .
$$

Then by the propernes of $f \times 1$ and irreducibility of $D_{k}(\tau)$, this set is closed and irreducible in $Y \times V$. If also $p_{2}$ denotes the projection of $Y \times V$ to $V$, then clearly 
$\overline{p_{2}\left(f \times 1\left(D_{k}(\tau)\right)\right)}=\overline{p_{2}\left(D_{k}(\tau)\right)}=\mathscr{W}$ and the map $\overline{p_{2}}: f \times 1\left(D_{k}(\tau)\right) \rightarrow \mathscr{W}$ is dominating. For $\psi \in \mathscr{W}$ with $X_{k}(\psi) \neq \varnothing$ then

$$
\operatorname{dim} f\left(X_{k}(\psi)\right)=\operatorname{dim}{\overline{p_{2}}}^{-1}(\psi) \geq \operatorname{dim} f \times 1\left(D_{k}(\tau)\right)-\operatorname{dim} \mathscr{W} .
$$

I claim that

$$
\begin{aligned}
& m_{k} \leq \operatorname{dim} Y \Rightarrow \operatorname{dim} D_{k}(\tau)=\operatorname{dim} f \times 1\left(D_{k}(\tau)\right) \\
& m_{k}>\operatorname{dim} Y \Rightarrow f \times 1\left(D_{k}(\tau)\right)=Y \times V .
\end{aligned}
$$

Let's see that the claim implies the theorem. Suppose $t_{k} \leq \operatorname{dim} Y$; then also $m_{k} \leq \operatorname{dim} Y$. If $X_{k}(\phi) \neq \emptyset$ then by the above

$$
\begin{aligned}
\operatorname{dim} f\left(X_{k}(\phi)\right) & \geq \operatorname{dim} f \times 1\left(D_{k}(\tau)\right)-\operatorname{dim} \mathscr{W} \\
& =\operatorname{dim} D_{k}(\tau)-\operatorname{dim} \mathscr{W} \\
& =t_{k} .
\end{aligned}
$$

Since $f\left(X_{k}(\phi)\right)$ is realized as a fiber of a certain dominating map, the inequality holds also for any irreducible component of $f\left(X_{k}(\phi)\right)$, by [10, I.8]. Hence part (1) of the theorem.

On the other hand suppose that $t_{k}>\operatorname{dim} Y$. Now if $m_{k} \leq \operatorname{dim} Y$ then since $X_{k}(0)=X$ is certainly non-empty, $\operatorname{dim} Y=\operatorname{dim} f\left(X_{k}(0)\right) \geq t_{k}$ as before, a contradiction. Hence we must have $m_{k}>Y$, so $f \times 1\left(D_{k}(\tau)\right)=Y \times V$. But then for any $\phi \in V$,

$$
f\left(X_{k}(\phi)\right)=p_{2}^{-1}(\phi)=Y,
$$

which is (2). Finally, (3) follows from the above and (A). So it remains to prove the claim. We first prove (B) which is easy: if $m_{k}>\operatorname{dim} Y$ then $\operatorname{dim} X-\operatorname{dim} Y>(e-k)(f-k)$. Let $p \in Y \times V$ be any point and consider the fiber $(f \times 1)^{-1} p$ over $p .(f \times 1)^{-1} p$ is projective, of dimension $\geq \operatorname{dim} X-\operatorname{dim} Y>(e-k)(f-k)$. But by assumption $p_{1}^{*}\left(E^{*} \otimes F\right)$ is ample on $(f \times 1)^{-1} p$, so the restriction of $\tau$ must drop rank according to the theorem of Fulton and Lazarsfeld [4, Th. 1.1]. That is, $D_{k}(\tau) \cap(f \times 1)^{-1} p \neq \emptyset$. Since $p$ was arbitrary, we conclude that $f \times 1\left(D_{k}(\tau)\right)=Y \times V$.

To prove (A), let $k^{\prime}=e-k$ and consider the Grassmanian $\pi: G:=$ $G_{k^{\prime}}\left(p_{1}^{*} E\right) \rightarrow X \times V$ with its tautological exact sequence

$$
0 \longrightarrow \mathscr{S} \longrightarrow \pi^{*} p_{1}^{*} E \longrightarrow \mathscr{Q} \longrightarrow 0 .
$$

Denote by $\sigma$ the composition $\mathscr{S} \hookrightarrow \pi^{*} p_{1}^{*} E \stackrel{\pi^{*} \tau}{\longrightarrow} \pi^{*} p_{1}^{*} F$. Then by Proposition 5, $\sigma$ is a regular section of $\mathscr{S}^{*} \otimes \pi^{*} p_{1}^{*} F$, and $Z(\sigma)$ is an irreducible variety. By construction $Z(\sigma)$ surjects onto $D_{k}(\tau)$. In fact, most concretely,

$$
Z(\sigma)=\{(x, \phi, H) \mid H \subset \operatorname{ker} \phi(x)\},
$$


and the map to $D_{k}(\tau)$ is just $(x, \phi, H) \mapsto(x, \phi)$. In this case the surjection is in fact birational, for the following reason. We need to find a point $(x, \phi) \in X \times V$ such that rank $\tau(x, \phi)$ is precisely equal to $k$, that is, $\operatorname{rank} \phi(x)=k$. But the map $V \longrightarrow\left(E^{*} \otimes F\right)(x) \longrightarrow 0$ is surjective so we just take any $\phi \in V$ mapping to a matrix of rank $k$.

Now we need to collect some open sets in $D_{k}(\tau)$. First, since $Z(\sigma) \rightarrow$ $D_{k}(\tau)$ is birational, there are open sets $U_{1}^{\prime} \subset Z(\sigma)$ and $U_{1} \subset D_{k}(\tau)$ with $U_{1}^{\prime} \cong U_{1}$. Next let $Y_{\text {sm }} \subset Y, X_{\text {sm }} \subset X$ be the open set of smooth points on $Y$ and $X$ and consider the map

$$
X_{\mathrm{sm}} \cap f^{-1}\left(Y_{\mathrm{sm}}\right) \longrightarrow Y_{\mathrm{sm}} .
$$

Since the map is dominant, by generic smoothness [8, III.10] there is an open set $U_{2} \subset X_{\text {sm }}$ so the tangent map

$$
T_{f}: T_{x} \longrightarrow T_{f(x)}
$$

is surjective for all $x \in U_{2}$ and $f(x)$ is smooth on $Y$. Notice that $(u, 0) \in$ $U_{2} \times V \cap D_{k}(\tau)$ for any $u \in U_{2}$ so $U_{2} \times V \cap D_{k}(\tau) \neq \emptyset$. Finally, there is of course an open set $U_{3} \subset D_{k}(\tau)$ such that for all $x \in U_{3}$, the image point $f \times 1(x)$ is smooth on $f \times 1\left(D_{k}(\tau)\right)$.

Now since $D_{k}(\tau)$ is irreducible, the open set

$$
U:=U_{1} \cap U_{2} \times V \cap U_{3} \subset D_{k}(\tau)
$$

is non-empty. Denote by $U^{\prime}$ the corresponding open set of $Z(\sigma)$. For any point $p \in U$ we then have: $f \times 1(p)$ is smooth on both $Y \times V$ and on $f \times 1\left(D_{k}(\tau)\right)$; the tangent map $T_{f \times 1}(p)$ has full rank and $p$ lies in the open set where $Z(\sigma) \rightarrow D_{k}(\tau)$ is an isomorphism.

Now assume, contrary to the claim, that $\operatorname{dim} f \times 1\left(D_{k}(\tau)\right)<\operatorname{dim} D_{k}(\tau)$. Look at the restriction

$$
U \hookrightarrow D_{k}(\tau) \longrightarrow f \times 1\left(D_{k}(\tau)\right) .
$$

This is a dominating map between irreducible varieties so by our assumption on the dimension of $f \times 1\left(D_{k}(\tau)\right)$ all non-empty fibers of the map are positive dimensional. Pick a point $p \in U$ and denote by $W$ an irreducible positive dimensional component of the fiber of $D_{k}(\tau) \rightarrow f \times 1\left(D_{k}(\tau)\right)$ over $f \times 1(p)$ that intersects $U$. Then $W$ is a complete variety, by the propernes of $f \times 1$. (Actually $W$ is projective because there is an ample line bundle on $W$, namely the determinant of $\left.\left.p_{1}^{*}\left(E^{*} \otimes F\right)\right|_{W}\right)$. Now the composition

$$
h:=f \times 1 \circ \pi: G \longrightarrow X \times V \longrightarrow Y \times V
$$


is proper, so we can pick a complete positive dimensional variety $W^{\prime}$ of $Z(\sigma)$ that intersects $U^{\prime}$ and maps birationally onto $W$. Pick any $g \in U^{\prime} \cap W^{\prime}$ and let $q=h(g)$. Consider the square:

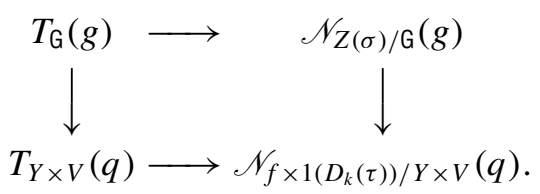

Here $\mathscr{N}$ denotes normal sheaves of the varieties $Z(\sigma)$ in $G$ and $f \times 1\left(D_{k}(\tau)\right)$ in $Y \times V$, respectively (the first is locally free by Prop. 5, and the second is locally free around $q$ by choice of $q$ ). Because the projection map $\pi: \mathrm{G} \rightarrow$ $X \times V$ is smooth, the tangent map $T_{\mathrm{G}}(g) \rightarrow T_{X \times V}(\pi(g))$ is surjective. And $T_{X \times V}(\pi(g)) \rightarrow T_{Y \times V}(q)$ is surjective by the choice of $U_{2}$. So the composition $T_{\mathrm{G}}(g) \rightarrow T_{Y \times V}(q)$ is surjective. Also $T_{Y \times V}(q) \rightarrow \mathscr{N}_{f \times 1\left(D_{k}(\tau)\right) / Y \times V}(q)$ is surjective because we chose $g$ such that $q$ is smooth both on $Y$ and on $f \times 1\left(D_{k}(\tau)\right)$. Summing up, the composition

$$
T_{\mathrm{G}}(g) \longrightarrow T_{Y \times V}(q) \longrightarrow \mathscr{N}_{f \times 1\left(D_{k}(\tau)\right) / Y \times V}(q)
$$

is surjective. Since by assumtion $f \times 1\left(D_{k}(\tau)\right)$ drops dimension, it can't be equal to $Y \times V$, so $\mathscr{N}_{f \times 1\left(D_{k}(\tau)\right) / Y \times V}(q) \neq 0$. Since the square commutes, to obtain a contradiction it is therefore enough to show that the composition of the other maps in the square is 0 .

To this end I claim that in fact the map of vector spaces

$$
\mathscr{N}_{Z(\sigma) / G}(g) \longrightarrow \mathscr{N}_{f \times 1\left(D_{k}(\tau)\right) / Y \times V}(q)
$$

is 0 . Now $W^{\prime} \subset h^{-1}(q)$ so by restricting $h^{*} \mathscr{N}_{f \times 1\left(D_{k}(\tau)\right) / Y \times V}$ to $W^{\prime}$ we obtain a trivial vector bundle; say $\left.h^{*} \mathscr{N}_{f \times 1\left(D_{k}(\tau)\right) / Y \times V}\right|_{W^{\prime}}=\mathscr{O}_{W^{\prime}}^{\oplus l}$ where $l=\operatorname{dim} Y \times V-$ $\operatorname{dim} f \times 1\left(D_{k}(\tau)\right)>0$. To prove that $\mathscr{N}_{Z(\sigma) / G}(g) \rightarrow \mathscr{N}_{f \times 1\left(D_{k}(\tau)\right) / Y \times V}(q)$ is 0 , it is clearly enough to prove that the map $\left.\left.\mathscr{N}_{Z(\sigma) / G}\right|_{W^{\prime}} \rightarrow h^{*} \mathscr{N}_{f \times 1\left(D_{k}(\tau)\right) / Y \times V}\right|_{W^{\prime}}=$ $\mathscr{O}_{W^{\prime}}^{\oplus l}$ is 0 . By Proposition 5, $\sigma$ is a regular section of $\mathscr{S}^{*} \otimes \pi^{*} p_{1}^{*} F$, hence

$$
\left.\mathscr{S}^{*} \otimes \pi^{*} p_{1}^{*} F\right|_{Z(\sigma)}=\mathscr{N}_{Z(\sigma) / G} .
$$

Note that since the map $\pi^{*} p_{1}^{*} E^{*} \longrightarrow \mathscr{S}^{*} \longrightarrow 0$ is surjective, also the map $\pi^{*}\left(p_{1}^{*} E^{*} \otimes p_{1}^{*} F\right) \longrightarrow \mathscr{S}^{*} \otimes \pi^{*} p_{1}^{*} F$ is surjective so to show the map

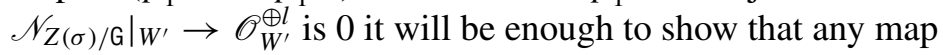

$$
\left.\pi^{*}\left(p_{1}^{*} E^{*} \otimes p_{1}^{*} F\right)\right|_{W^{\prime}} \longrightarrow \mathscr{O}_{W^{\prime}}^{\oplus l}
$$

is in fact 0 . By assumption $\left.p_{1}^{*}\left(E^{*} \otimes F\right)\right|_{W}$ is an ample vector bundle and $\pi: W^{\prime} \rightarrow W$ is a birational morphism of complete varieties so this fact follows from Lemma 3. This concludes the proof of Theorem 7. 
REMARK 3. The proof of the theorem goes through in arbitrary characteristic if in addition to the hypothesis of Theorem 7 one assumes that the map $f$ is generically smooth.

COROLlaRY 8. Suppose $X$ is a projective variety (over a field of arbitrary characteristic) and $F$ an ample, globally generated vector bundle on $X$ of $\operatorname{rank} F \geq \operatorname{dim} X$. Then there is a section $\sigma \in \Gamma(X, F)$ such that $Z(\sigma)$ is a finite non-empty set of points.

Proof. The structure map $X \rightarrow \operatorname{Spec} K$ (where $K$ is the ground field) is generically smooth so by Remark 3, Theorem 7 applies with $Y=\operatorname{Spec} K$, $E=\mathscr{O}_{X}$ and $k=0$. By Theorem 7 (3) we have $m_{0} \leq 0 \Rightarrow t_{0} \leq 0$. Now $m_{0}=\operatorname{dim} X-\operatorname{rank} F$, and $t_{0} \leq 0$ means precisely that there exist a section as stated.

Sub-Corollary 9 ([2]). Suppose $X$ is a projective variety of dimension $n$ and $F$ an ample, globally generated vector bundle on $X$, of rank greater than or equal to $n$. Then $c_{n}(F)>0$.

Proof. If rank $F=n$, pick a section $\sigma \in \Gamma(X, F)$ which vanishes at finitely many points. Then $c_{n}(F)$ is represented by a positive cycle with support on $Z(\sigma)$ [3, Prop. 14.1]. If rank $F>n$, pick a trivial line subbundle $\mathscr{O}_{X}$ of $F$ and let $\mathscr{Q}$ be the quotient of $F$ by $\mathscr{O}_{X}$. Then $c_{n}(\mathscr{Q})=c_{n}(F)$ and $\mathscr{Q}$ is globally generated and ample, so induction finishes the proof.

In fact, in the next section we will see how the ideas of the proof of Theorem 7 give an explicit formula for $c_{n}(F)$. For the rest of this section we will give some examples to illustrate Theorem 7.

Example 3. Referring back to Example 2 in $\S 1$, let $A^{1} \times$ pt be a section of $p_{2}^{*} \mathscr{O}(1)$. Then $p_{1}\left(\mathrm{~A}^{1} \times \mathrm{pt}\right)=\mathrm{A}^{1}$ doesn't drop dimension, as predicted by Theorem 7. But $p_{2}\left(\mathrm{~A}^{1} \times \mathrm{pt}\right)=\mathrm{pt}$ drops dimension. In the latter case, however, $p_{2}$ is not a proper morphism (in fact it is affine).

In Example 1, the exceptional divisor $E$ is a section of the bundle $\mathscr{O}_{X}(E)$ that drops dimension under the projection map $\pi$ ( $E$ is mapped to a point). But as we saw, $\mathscr{O}_{X}(E)$ isn't ample rel $\pi$.

EXAmple 4. Theorem 7 is optimal, in the following sense. It says that the generic degeneracy locus doesn't drop dimension, so one could ask if in fact no $X_{k}(\phi)$ drops dimension. However this is not true. For example, in Example 2 , let $x$ be the coordinate on $\mathrm{A}^{1}$ and $(s: t)$ coordinates on $\mathrm{P}^{1}$ and consider the section

$$
\sigma=(s x, t x) \in \Gamma\left(\mathrm{A}^{1} \times \mathrm{P}^{1}, p_{2}^{*} \mathscr{O}(1) \oplus p_{2}^{*} \mathscr{O}(1)\right) .
$$

Then $Z(\sigma)=\{0\} \times \mathrm{P}^{1}$ so $\operatorname{dim} Z(\sigma)=1$. On the other hand, $p_{1}(Z(\sigma))=\{0\}$ has dimension 0 . 
EXAMPLE 5. Theorem 7 fails without the globally generated hypothesis. Let $C$ be a non-hyperelliptic curve of genus $g>2$. Pick two different points $P, Q$ on $C$ and consider the divisor $D:=2 P-Q$. Let $\mathscr{L}=\mathscr{O}(D)$. Then $H^{0}(C, \mathscr{L})=0$ because $C$ is non-hyperelliptic. And $\mathscr{L}$ is ample because its degree is $1>0$. I claim there is a rank 2 vector bundle $F$ on $C$ and a non-trivial extension

$$
0 \longrightarrow \mathscr{O}_{C} \longrightarrow F \longrightarrow \mathscr{L} \longrightarrow 0
$$

Now $\operatorname{Ext}^{1}\left(\mathscr{L}, \mathscr{O}_{C}\right) \cong H^{1}\left(C, \mathscr{L}^{*}\right)$ and this group can be calculated from Riemann-Roch:

$$
h^{0}\left(C, \mathscr{L}^{*}\right)-h^{1}\left(C, \mathscr{L}^{*}\right)=-1+1-g=-g .
$$

Since $\mathscr{L}$ is ample, $h^{0}\left(C, \mathscr{L}^{*}\right)=0$, so this reads $h^{1}\left(C, \mathscr{L}^{*}\right)=g>0$. So we can pick a non-trivial extension $(*)$ corresponding to a non-zero element of $\operatorname{Ext}^{1}\left(\mathscr{L}, \mathscr{O}_{C}\right)$. According to Gieseker's theorem 2.2 [5] $F$ is an ample vector bundle. $F$ has a trivial subbundle by $(*)$ and hence a nowhere vanishing section 1. But $H^{0}(C, F)=\mathrm{C} \cdot 1$ by the long exact sequence of cohomology coming from $(*)$, since $\mathscr{L}$ has no sections. Hence the only global section of $F$ that does vanish somewhere is the zero-section, which vanishes everywhere. So the number $t_{0}=\operatorname{dim} C=1$ in this case. Consider the projection $f: C \rightarrow \operatorname{Spec} C$ of the curve to the ground field. If Theorem 7 (2) was true without the globally generated hypothesis, then for any section $\sigma \in \Gamma(C, F), f(Z(\sigma))=$ Spec C; in other words every section of $F$ should vanish somewhere. But as we have seen, $F$ has a nowhere vanishing section, so this is a contradiction. In this case, $m_{0}=-1 \leq 0=\operatorname{dim} Y<t_{0}$ so statement (3) also fails.

This example proves that the second and third statement of Theorem 7 do not hold if one omits the globally generated hypothesis. Modifying the example a bit, it also gives a counterexample to the first statement, for a non-globally generated bundle. Namely, consider the variety $C \times A^{1}$ with its two projection maps

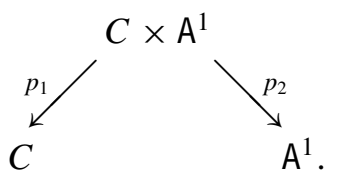

The bundle $p_{1}^{*}(F)$ is ample rel $p_{2}$. If $x$ denotes the coordinate on $A^{1}$ then $H^{0}\left(C \times \mathrm{A}^{1}, p_{1}^{*}(F)\right)=H^{0}(C, F) \oplus x \cdot H^{0}(C, F) \oplus x^{2} \cdot H^{0}(C, F) \oplus \ldots$ But since $H^{0}(C, F)=\mathrm{C} \cdot 1$, this can be identified with $\mathrm{C}[x]$. Hence if $\sigma=P(x) \in$ $H^{0}\left(C \times \mathrm{A}^{1}, p_{1}^{*}(F)\right)$ is a section, and $x_{1}, x_{2}, \ldots, x_{n}$ denotes the roots of $P(x)$, then $Z(\sigma)=C \times\left\{x_{1}\right\} \cup C \times\left\{x_{2}\right\} \cup \ldots \cup C \times\left\{x_{n}\right\}$. Therefore, all sections 
that do vanish somewhere vanish in dimension 1 , so $t_{0}=1$. Also note that $p_{2}(Z(\sigma))=\left\{x_{1}, x_{2}, \ldots, x_{n}\right\}$ is a finite set of points.

Now consider the proper surjective map $p_{2}: C \times \mathrm{A}^{1} \rightarrow \mathrm{A}^{1}$. If Theorem 7 (1) was correct without the globally generated hypothesis then $\operatorname{dim} p_{2}(Z(\sigma)) \geq 1$ for any section $\sigma \in H^{0}\left(C \times \mathrm{A}^{1}, p_{1}^{*}(F)\right)$. But $p_{2}(Z(\sigma))$ is a finite set of points for all non-zero $\sigma$, which is a contradiction.

\section{Globally generated bundles of high rank}

Let $X$ be any projective variety (over an algebraically closed field of arbitrary characteristic) and $E$ a globally generated vector bundle on $X$. Let $n=\operatorname{dim} X$ and $e=\operatorname{rank} E$. Our purpose is to show how some of the ideas that prove Theorem 7 give an explicit formula for $c_{n}(E)$ in case $E$ has a section that vanishes on a finite set of points (for instance when $E$ is ample). We start with

Proposition 10. Suppose E is a globally generated bundle on the projective variety $X$ of dimension $n$. Then

(1) $c_{n}(E) \geq 0$;

(2) $c_{n}(E)>0 \Longleftrightarrow \exists \sigma \in \Gamma(X, E): Z(\sigma)$ is a finite non-empty set of points.

Proof. Let $V=\Gamma(X, E)$ be a finite dimensional space of sections that generate $E$. Then there is an exact sequence

$$
0 \rightarrow \mathscr{K} \longrightarrow \mathscr{O}_{X} \otimes V \longrightarrow E \rightarrow 0 .
$$

Consider $X \times V$ with its two projections $p_{1}, p_{2}$ to $X$ resp. $V$. The tautological section $\sigma_{t} \in \Gamma\left(X \times V, p_{1}^{*}\left(\mathscr{O}_{X} \otimes V\right)\right)$ maps to a section $\sigma$ of $p_{1}^{*}(E)$ whose zero-set is the incidence correspondance

$$
Z(\sigma)=\{(x, s) \mid s(x)=0\} \subset X \times V .
$$

Lemma 6 shows that $Z(\sigma)$ can be considered as an affine space bundle over $X$, in fact

$$
Z(\sigma)=\operatorname{Spec} \operatorname{Sym} \mathscr{K}^{*} .
$$

Note that the image of $Z(\sigma)$ under $p_{2}$ is the cone $C:=\{s \in V \mid Z(s) \neq$ $\emptyset\}$ of sections with non-empty zero-scheme. We now projectivize the whole situation. Thus we get a diagram

$$
\begin{aligned}
\operatorname{Proj} \operatorname{Sym} \mathscr{K}^{*} & =\{(x,[s] \mid s(x)=0\} \subset X \times \mathrm{P} V \\
p_{2} \downarrow & \\
\mathrm{PC} & =\{[s] \mid Z(s) \neq \emptyset\} \subset \mathrm{P} V .
\end{aligned}
$$


We let $\mathrm{P} K=$ Proj Sym $\mathscr{K}^{*}$. There is a tautological line bundle $\mathscr{O}_{\mathrm{P} K}(1)$ on $\mathrm{P} K$; this is the restriction to $\mathrm{P} K$ of the line bundle $\mathscr{O}_{X \times \mathrm{PV}}(1)=p_{2}^{*} \mathscr{O}_{\mathrm{P} V}(1)$. By the definition of Segre classes [3, Chap. 3] we have

$$
\begin{aligned}
s_{n}(\mathscr{K}) & =\int_{\mathrm{P} K} c_{1}\left(\mathscr{O}_{\mathrm{P} K}(1)\right)^{\operatorname{dim} \mathrm{P} K} \\
& =\int_{X \times \mathrm{PV}} c_{1}\left(p_{2}^{*} \mathscr{O}_{\mathrm{P} V}(1)\right)^{\operatorname{dim} \mathrm{P} K} \cap[\mathrm{P} K] \\
& =\int_{\mathrm{P} V} c_{1}\left(\mathscr{O}_{\mathrm{P} V}(1)\right)^{\operatorname{dim} \mathrm{P} K} \cap p_{2 *}[\mathrm{P} K] ;
\end{aligned}
$$

where the last equality is the projection formula for the proper mapping $p_{2}$. But the defining exact sequence of $\mathscr{K}$ reveals that $s_{n}(\mathscr{K})=c_{n}(E)$, hence

$$
c_{n}(E)=\int_{\mathrm{P} V} c_{1}\left(\mathscr{O}_{\mathrm{PV}}(1)\right)^{\operatorname{dim} \mathrm{P} K} \cap p_{2 *}[\mathrm{P} K]
$$

which immediately proves (1). Also the formula reveals that $c_{n}(E)$ is nonzero precisely when $p_{2 *}[\mathrm{P} K]$ is non-zero, i.e. when $\operatorname{dim} \mathrm{P} K=\operatorname{dim} p_{2}(\mathrm{P} K)$. Now $p_{2}(\mathrm{P} K)=\mathrm{PC}$ and the dimensions are the same precisely when the map $\mathrm{P} K \rightarrow \mathrm{PC}$ has a fiber consisting of a finite non-empty set of points. But for every $[s] \in \mathrm{P} C$, the fiber over $[s]$ identifies with the zero-scheme of $s$, which proves (2).

Now suppose $E$ has a section which vanishes at a finite set of points - or equivalently, that $c_{n}(E)>0$. Then in the notation of the proof of Proposition $10, p_{2 *}[\mathrm{P} K]=\operatorname{deg} p_{2} \cdot[\mathrm{P} C]$, and the degree of $p_{2}: \mathrm{P} K \rightarrow \mathrm{PC}$ is just the length of a generic fiber, i.e. the length of the subscheme $Z(s)$ for a generic $s \in C$. We call this number $l$. Then since

$$
\int_{\mathrm{P} V} c_{1}\left(\mathscr{O}_{\mathrm{PV}}(1)\right)^{\operatorname{dim} \mathrm{PC}} \cap[\mathrm{PC}]=\operatorname{deg} \mathrm{PC},
$$

we get the formula alluded to in the introduction:

Formula 11. Suppose $E$ is a globally generated bundle of rank $e$ on the projective variety $X$, of dimension $n$. Suppose $E$ has a section that vanishes at finitely many points (or equivalently that $c_{n}(E)>0$ ). Let $C:=\{s \in$ $\Gamma(X, E) \mid Z(s) \neq \emptyset\}$ be the cone of sections that vanish somewhere. Then $C$ is an irreducible subvariety of $\Gamma(X, E)$ and $\operatorname{codim}_{\Gamma(X, E)} C=e-n$. If $l$ denotes the length of the subscheme $Z(s)$ for a generic section $s$ in $C$ then

$$
c_{n}(E)=l \cdot \operatorname{deg} C .
$$


EXAMPLE 6. The formula applies if for instance $E$ is globally generated and ample, as follows from Corollary 8. In general, let $E$ be a globally generated vector bundle of rank $e$ on the projective variety $X$. Let $v=\operatorname{dim} \Gamma(X, E)$ be the dimension of the space of global sections of $E$. Then $E$ defines a map $g: X \rightarrow G_{v-e}(\Gamma(X, E))$ to the grassmanian of $v-e$-planes in $\Gamma(X, E)$. If the rank of $E$ is at least equal to the dimension of $X$, and there is a smooth point on $X$ where the tangent map of $g$ is injective, then one can show much in the spirit of [5, Lemma 1.1] that $E$ has a section which vanishes at finitely many points. At least in characteristic 0 , this condition is weaker than ampleness, since if $E$ is ample $g$ must neccesarily have finite fibers so by generic smoothness there exists a smooth point on $X$ where the tangent map of $g: X \rightarrow g(X)$ is surjective, hence injective. Then Formula 11 applies to the top Chern class of E.

EXAmple 7. If $E$ is "sufficiently positive" - for instance, if $E(-1)$ is globally generated for some very ample line bundle $\mathscr{O}_{X}(1)$ (which in particular implies that $E$ is globally generated and ample) - and $\operatorname{rank} E>\operatorname{dim} X$, then the number $l$ in Formula 11 is 1 . To see this one simply counts the number of sections of $E$ that vanish at a point $x \in X$, and then the number of sections that vanish at $x$ and at another point of $X$, using that since $E$ is a quotient of a direct sum of very ample line bundles, the evaluation map

$$
\Gamma(X, E) \rightarrow \Gamma\left(E \otimes \mathscr{O}_{Z}\right)
$$

is surjective for any length 2 subscheme $Z$ of $X$.

EXAMPLE 8 . For an arbitrary ample, globally generated bundle $l$ might be bigger than 1 . In fact, $l$ might take any positive integer as value. To see this, note that for $g=2 d-2$, and $r=1$, the Brill-Noether number $\rho(g, r, d):=$ $g-(r+1)(g+r-d)$ is zero, and takes the value -2 if $d$ is substituted by $d-1$. By the theorems of Kempf, Kleiman-Laksov (see e.g. [4]) and GriffithHarris [6], it follows that every curve of genus $2 d-2$ has a linear system of degree $d$ and dimension 1 (a $g_{d}^{1}$ ) but the general curve of this genus has no $g_{d-1}^{1}$. Letting $X$ be a general curve of genus $2 d-2$, the linear system $g_{d}^{1}$ must then neccesarily be base-point free, hence the corresponding line bundle $\mathscr{L}_{d}$ is globally generated (and ample since deg $\mathscr{L}_{d}=d>0$ ). Let $E_{d}:=\mathscr{L}_{d} \oplus \mathscr{L}_{d}$, then $E_{d}$ is an ample and globally generated bundle on $X$. By construction, $X$ has no $g_{d-1}^{1}$, so if two divisors in $g_{d}^{1}$ have a point in common, they must be equal. This means that for $E_{d}$, the number $l$ is equal to $d$, and $d$ can be any positive integer. In this case $c_{1}\left(E_{d}\right)=2 d$, so Formula 11 reads $2 d=d \cdot 2$. Hence the cone $C$ is a quadric hypersurface in the space of global sections of E. 
We suppose now that $E$ is any bundle on a projective variety $X$ such that $E$ satisfies the conditions in Formula 11. If rank $E=\operatorname{dim} X:=n$, then $c_{n}(E)=l$ so by Formula 11 we see that $\operatorname{deg} C=1$ and $\operatorname{codim} C=0$, i.e. $C=\Gamma(X, E)$ so all sections of $E$ must vanish. If rank $E>\operatorname{dim} X$ then $\operatorname{deg} C=1$ means that $C$ is linear, hence has a complement $C^{\prime}$ inside $\Gamma(X, E)$ so $\Gamma(X, E)=C \oplus C^{\prime}$. But then the defining sequence of $E$ becomes

$$
0 \rightarrow \mathscr{K} \longrightarrow \mathscr{O}_{X} \otimes\left(C \oplus C^{\prime}\right) \longrightarrow E \rightarrow 0
$$

and it is clear that $\mathscr{K}$ is contained in the summand $\mathscr{O}_{X} \otimes C$. Hence $E \cong$ $\left(\mathscr{O}_{X} \otimes C\right) / \mathscr{K} \bigoplus \mathscr{O}_{X} \otimes C^{\prime}$ so in particular $E$ has a trivial direct summand. If we let $E^{\prime}=\left(\mathscr{O}_{X} \otimes C\right) / \mathscr{K}$ and $E^{\prime \prime}=\mathscr{O}_{X} \otimes C^{\prime}$ so that $E \cong E^{\prime} \oplus E^{\prime \prime}$, then $E^{\prime}$ has rank $n=\operatorname{dim} X$, and we have a good knowledge of $E$ : it is a direct sum of a vector bundle $E^{\prime}$ of $\operatorname{rank} \operatorname{dim} X$, for which all sections vanish, with a trivial bundle. The same argument shows that more generally, if one just assumes $C$ degenerate - i.e. contained in some linear space $\neq \Gamma(X, E)$ - then $E$ has a trivial direct summand. But if for instance $E$ is ample, it cannot contain a trivial direct summand. We mention two applications of this idea:

COROLlaRY 12. Let $E$ be a globally generated, ample vector bundle on a projective variety $X$ such that $\operatorname{rank} E>\operatorname{dim} X:=n$. Then if $c_{n}(E)$ is a prime number, $E$ has a section which vanishes at exactly one point.

Corollary 13 (due to L. Manivel). Let E be a globally generated, ample vector bundle of rank e on a projective variety $X$ of dimension $n$. Then $c_{n}(E) \geq$ $e-n+1$.

PRoofs. Corollary 12 follows from Corollary 8 and Formula 11 in view of the fact that $\operatorname{deg} C>1$ since $E$ is ample. As for Corollary 13 we may of course assume that $e \geq n$. Then by Corollary $8 E$ has a section which vanishes at finitely many points, so Formula 11 applies to show that $c_{n}(E) \geq \operatorname{deg} C=$ $\operatorname{deg} \mathrm{PC}$. Since $E$ is ample, $\mathrm{P} C$ is non-degenerate, so the degree of $\mathrm{P} C$ is at least equal to its codimension +1 [9, Cor. 18.12], which again by Formula 11 is $e-n+1$.

The conjecture of Ballico [1] states that the inequality in Corollary 13 can be improved to $c_{n}(E) \geq\left(\begin{array}{l}e \\ n\end{array}\right)$. For curves, this gives the lower bound $e$, coinciding with the bound found in Corollary 13, but for higher dimensional varieties Ballico's conjectural bound is of course much stronger than the bound in Corollary 13. Ballico's conjecture is known to be true if one imposes certain further positivity conditions on the bundle - for instance, if one asks $E(-1)$ globally generated for some very ample line bundle $\mathscr{O}(1)$, Ballico's inequality follows readily from Proposition 10 and [3, Example 3.2.2] - but is unknown 
for a merely ample and globally generated bundle. In this respect, Corollary 13 apparently gives the first non-trivial bound valid for any ample, globally generated bundle on a projective variety of arbitrary dimension.

\section{REFERENCES}

1. Ballico, E., Spanned and Ample Vector Bundles with Low Chern Numbers, Pacific J. Math. 140 (1989), 209-216.

2. Bloch, S., Gieseker, D., The Positivity of the Chern Classes of an Ample Vector Bundle, Invent. Math. 12 (1971), 112-117.

3. Fulton, W., Intersection Theory, Ergeb. Math. Grenzgeb. 2 (1984).

4. Fulton, W., Lazarsfeld, R., On the Connectedness of Degeneracy Loci and Special Divisors, Acta Math. 146 (1981), 271-283.

5. Gieseker, D., p-Ample Bundles and their Chern Classes, Nagoya Math. J. 43 (1971), 91-116.

6. Griffiths, P., Harris, J., On the Variety of Special Linear Systems on a General Algebraic Curve, Duke Math. J. 47 (1980), 233-272.

7. Hartshorne, R., Ample Vector Bundles, Inst. Hautes Études Sci. Publ. Math. 29 (1966), 63-94.

8. Hartshorne, R., Algebraic Geometry, Graduate Texts in Math. 52 (1977).

9. Harris, J., Algebraic Geometry, Graduate Texts in Math. 133 (1992).

10. Mumford, D., The Red Book of Varieties and Schemes, Lecture Notes in Math. 1358 (1988).

11. Steffen, F., A Generalized Principal Ideal Theorem with an Application to Brill-Noether Theory, Invent. Math. 132 (1998), 73-90.

LABORATOIRE J. A. DIEUDONNÉ

PARC VALROSE

06108 NICE

FRANCE

E-mail: geertsen@math.unice.fr
CURRENT ADDRESS:

DANSKE BANK

BUSINESS AND IT INNOVATION

SHARED SERVICES CENTRE

HOMENS KANAL 2-12

DK-1092 COPENHAGEN K

E-mail: andersg@danskebank.dk 